\title{
EXTENDING THE TAMARI LATTICE TO SOME COMPOSITIONS OF SPECIES
}

\author{
STEFAN FORCEY
}

\begin{abstract}
An extension of the Tamari lattice to the multiplihedra is discussed, along with projections to the composihedra and the Boolean lattice. The multiplihedra and composihedra are sequences of polytopes that arose in algebraic topology and category theory. Here we describe them in terms of the composition of combinatorial species. We define lattice structures on their vertices, indexed by painted trees, which are extensions of the Tamari lattice and projections of the weak order on the permutations. The projections from the weak order to the Tamari lattice and the Boolean lattice are shown to be different from the classical ones. We review how lattice structures often interact with the Hopf algebra structures, following Aguiar and Sottile who discovered the applications of Möbius inversion on the Tamari lattice to the Loday-Ronco Hopf algebra.
\end{abstract}

\section{INTRODUCTION}

We will be looking at the following spectrum of polytopes:
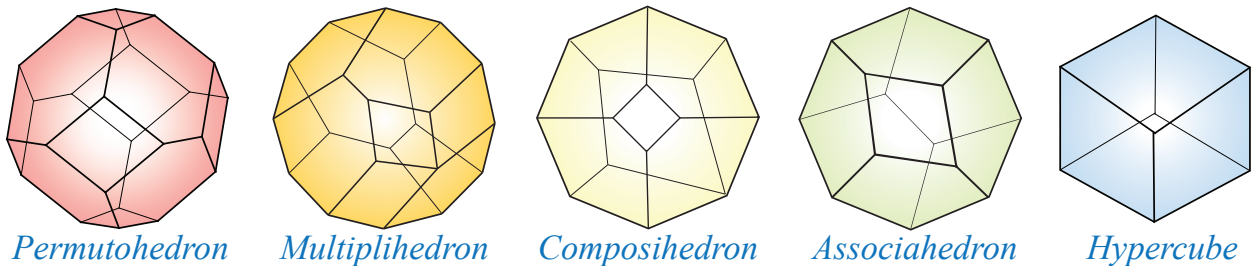

Here is a planar rooted binary tree, often called a binary tree:
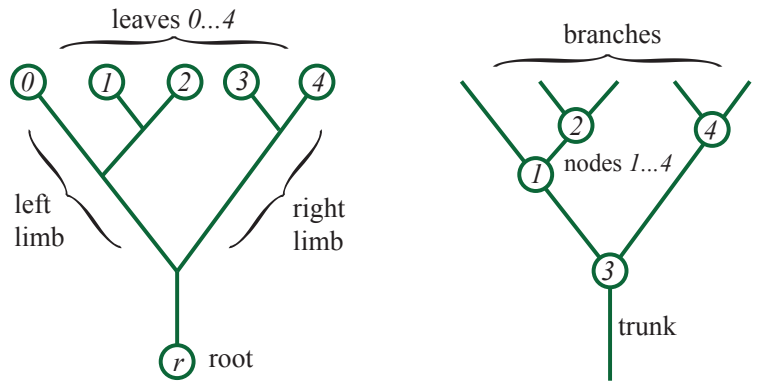

We only label the leaves and interior nodes (branch points) with their left-to-right ordering when necessary. The branches are the edges with a leaf. The nodes are also partially ordered by their proximity to the root, which is maximal; in the picture node $3>1>2$. The set of planar rooted binary trees with $n$ nodes and $n+1$ leaves is denoted $\mathcal{Y}_{n}$. 
1.0.1. Notation. We will choose from the current rather prolix notation used for the classical polytopes and lattices, and try to decrease the proliferation of symbols by referring to a polytope and its associated orders by a common name. The context will determine whether we are focused on the face structure, the vertices alone, or the 1-skeleton. Since the vertices are used more often, we let that be the default meaning, and add more specifics if necessary. For instance if we wish to refer to the general planar trees that index the faces of the associahedron, we'll make that clear.

The Tamari lattice is denoted either $\mathcal{Y}_{n}$ or $\mathbb{T}_{n}$. The set of painted trees with $n$ nodes and $n+1$ leaves is denoted $\mathcal{M}_{n}$ and the lattice structure on that set is denoted $\mathcal{M}_{n}$ as well. The set of binary trees with leaves weighted by positive integers summing to $n+1$ is denoted $\mathcal{C} \mathcal{K}_{n}$ and the lattice structure we define on that set is denoted $\mathcal{C} \mathcal{K}_{n}$ as well. Let $[n]=\{1, \ldots, n\}$. The Boolean lattice of subsets of $[n]$ is denoted $\mathcal{Q}_{n}$. The lattice of weakly ordered permutations on $[n]$, described below, is denoted $\mathfrak{S}_{n}$. We also continue this abusive notation by using the same symbols to denote the polytopes whose vertices are indexed by the indicated set. Thus the $(n-1)$-dimensional associahedron is denoted $\mathcal{Y}_{n}$, which corresponds to the notation $\mathcal{K}_{n}$ in 13 or $\mathcal{K}(n+1)$ in 7 or even $K_{n+1}$ in Stasheff's original notation. The $(n-1)$-dimensional permutohedron, multiplihedron, composihedron and hypercube are denoted $\mathfrak{S}_{n}, \mathcal{M}_{n}, \mathcal{C K}_{n}$, and $\mathcal{Q}_{n}$ respectively. Rather than a subscript $n$, we sometimes use a placeholder $\bullet$ to refer to the entire sequence at once.

The 1-skeleta of the families of polytopes $\mathfrak{S}_{.}, \mathcal{M} ., \mathcal{Y}$. and $\mathcal{Q}$. are Hasse diagrams of posets. For the permutahedron $\mathfrak{S}_{n}$, the corresponding poset is the (left) weak order, which we describe in terms of permutations. A cover in the weak order has the form $w \lessdot(k, k+1) w$, where $k$ precedes $k+1$ among the values of $w$. Figure 1 displays the weak order on $\mathfrak{S}_{4}$, the Tamari order on $\mathcal{Y}_{4}$ and the Boolean lattice $\mathcal{Q}_{3}$.

1.1. Species. A combinatorial species of sets is an endofunctor of Finite Sets with bijections.

Example 1.1. The species $\mathcal{L}$ of lists takes a set to linear orders of that set.

$\mathcal{L}(\{a, d, h\})=\{a<d<h, a<h<d, h<a<d, h<d<a, d<a<h, d<h<a\}$

Example 1.2. The species $\mathcal{Y}$ of binary trees takes a set to trees with labeled leaves.

$$
\mathcal{Y}(\{a, d, h\})=\left\{Y^{a} y^{h}, Y^{h d}, \ldots, Y^{a d}, Y^{h d}, \ldots\right\}
$$

We define the composition of two species following Joyal in [12]:

$$
(\mathcal{G} \circ \mathcal{H})(U)=\bigsqcup_{\pi} \mathcal{G}(\pi) \times \prod_{U_{i} \in \pi} \mathcal{H}\left(U_{i}\right)
$$

where the union is over partitions of $U$ into any number of nonempty disjoint parts.

$$
\pi=\left\{U_{1}, U_{2}, \ldots, U_{n}\right\} \text { such that } U_{1} \sqcup \cdots \sqcup U_{n}=U \text {. }
$$



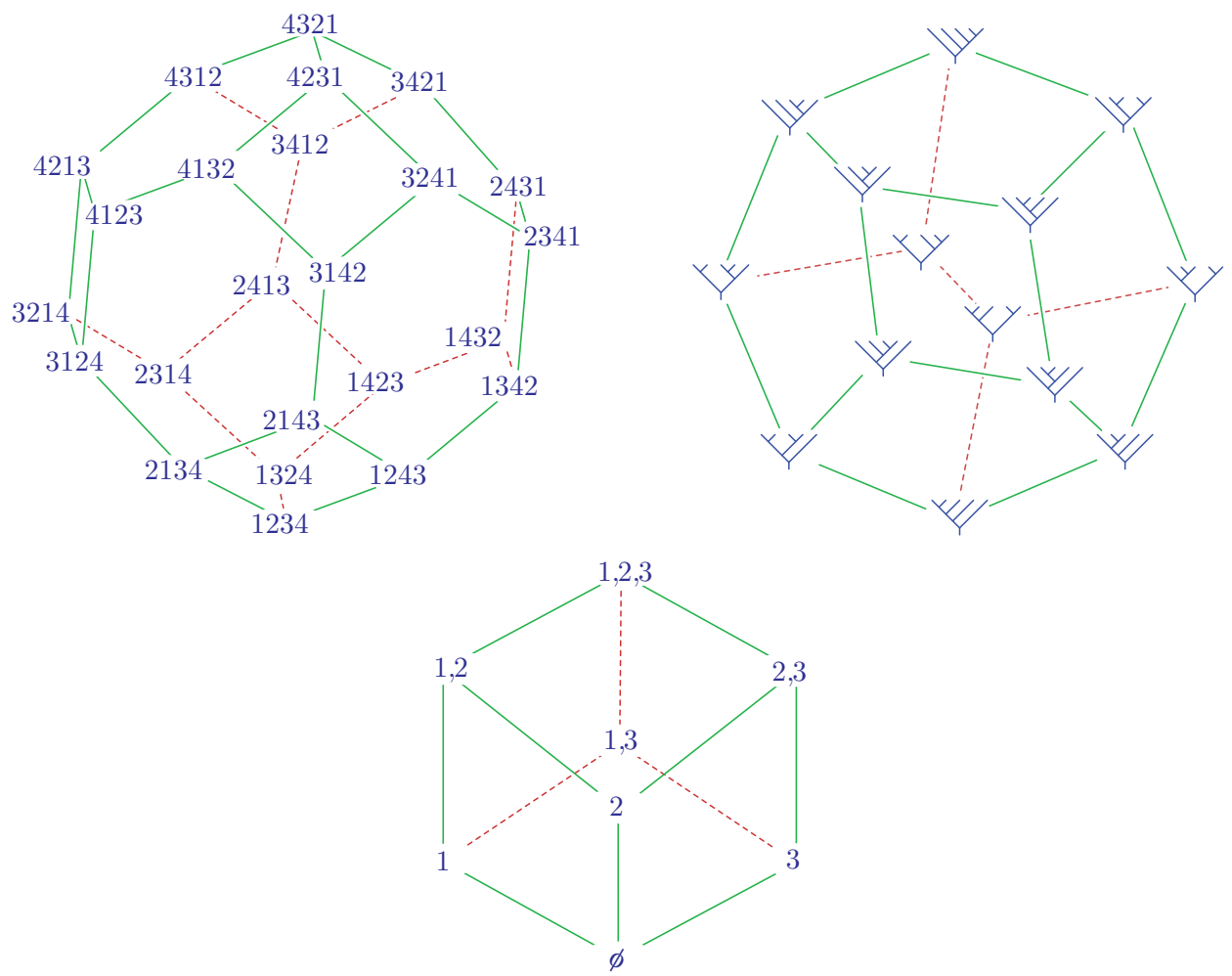

Figure 1. Hasse diagrams of three classical lattices in this paper:

Weak order on $\mathfrak{S}_{4}$, Tamari lattice $\mathcal{Y}_{4}$ and Boolean lattice $\mathcal{Q}_{3}$.

This formula also appears to be known as the cumulant formula, the moment sequence of a random variable, and the domain for operad composition:

$$
\gamma: \mathcal{F} \circ \mathcal{F} \rightarrow \mathcal{F} \text {. }
$$

\section{SEVERAL Flavors of TREeS}

2.1. Ordered, Bi-leveled and Painted trees. Many variations of the idea of the binary tree have proven useful in applications to algebra and topology. Each variation we mention can have its leaves labeled, providing an example of a set species.

An ordered tree (sometimes called leveled) has a vertical ordering of the $n$ nodes as well as horizontal. This allows a well-known bijection between the ordered trees with $n$ nodes and the permutations $\mathfrak{S}_{n}$. We will call this bijection $b i j_{1}$. This bijection and all the other maps we will discuss are demonstrated in Figure 7 .

As defined in 2.1 of [9], a bi-leveled tree $(t ; \mathrm{T})$ is a planar binary tree $t \in \mathcal{Y}_{n}$ together with an (upper) order ideal $\mathrm{T}$ of its node poset, where $\mathrm{T}$ contains the leftmost node of $t$ as a minimal element. (Recall that an upper order ideal is a sub-poset such that $x>y \in \mathrm{T}$ implies $x \in \mathrm{T}$.) We draw the underlying tree $t$ and circle the nodes in $\mathrm{T}$. By the condition on $\mathrm{T}$, all nodes along the leftmost branch are circled and none are circled above the leftmost node. 
Saneblidze and Umble [17] introduced bi-leveled trees in terms of equivalence classes on ordered trees. They describe a cellular projection from the permutahedra to Stasheff's multiplihedra $\mathcal{M}$., with the bi-leveled trees on $n$ nodes indexing the vertices $\mathcal{M}_{n}$.

Definition 2.1. We denote Saneblidze and Umble's map as $\beta: \mathfrak{S}_{n} \rightarrow \mathcal{M}_{n}$, as in [9], and describe it as the map which first circles all the nodes vertically ordered below and including the leftmost node, and then forgets the vertical ordering of the nodes.

Numbering the nodes in a tree $t \in \mathcal{Y}_{n} 1, \ldots, n$ from left to right, $\mathrm{T}$ becomes a subset of $\{1, \ldots, n\}$.

Definition 2.2. The partial order on $\mathcal{M}_{n}$ is defined by $(s ; \mathrm{S}) \leq(t ; \mathrm{T})$ if $s \leq t$ in $\mathcal{Y}_{n}$ and $\mathrm{T} \subseteq \mathrm{S}$.

Theorem 2.3. The poset of bi-leveled trees is a lattice.

Proof. The unique supremum of two bi-leveled trees $(t ; \mathrm{T})$ and $(s ; \mathrm{S})$ is found by first taking their unique supremum $\sup \{t, s\}$ in the Tamari lattice, and then circling as many nodes of $\sup \{t, s\}$ in the intersection $\mathrm{T} \cap \mathrm{S}$ as are allowed by the upper order ideal condition. That is, the circled nodes of the join comprise the largest order ideal of nodes of $\sup \{t, s\}$ that is contained in $\mathrm{T} \cap \mathrm{S}$. The unique infimum is found by taking the infimum of the two trees in the Tamari lattice, the union of the two order ideals, and adding to the latter any nodes necessary to make that union an order ideal in the node poset of $\inf \{s, t\}$. That is, the meet is given by $(\inf \{t, s\} ; \mathrm{T} \cup \mathrm{S} \cup\{x \mid x>y \in \mathrm{T} \cup \mathrm{S}\})$.

The Hasse diagrams of the posets $\mathcal{M}_{n}$ are 1-skeleta for the multiplihedra. The Hasse diagram of $\mathcal{M}_{4}$ appears in Figure 4. Stasheff used a different type of tree for the vertices of $\mathcal{M}$.. A painted binary tree is a planar binary tree $t$, together with a (possibly empty) upper order ideal of the node poset of $t$. (Recall the root node is maximal.) We indicate this ideal by painting part of a representation of $t$. For clarity, we stop our painting in the middle of edges (not precisely at nodes). Here are a few simple examples,

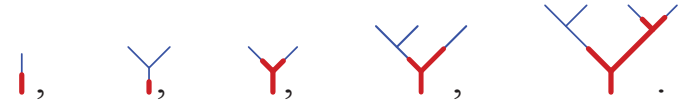

An $A_{n}$-space is a topological $H$-space with a weakly associative multiplication of points [18. Maps between $A_{\infty}$ spaces are only required to preserve the $A_{\infty}$ structure up to homotopy. Stasheff [19] described these maps combinatorially using cell complexes called multiplihedra, while Boardman and Vogt [4] used spaces of painted trees. Both the spaces of trees and the cell complexes are homeomorphic to convex polytope realizations of the multiplihedra as shown in 7 .

If $f:(X, \bullet) \rightarrow(Y, *)$ is an $A_{\infty}$-map homotopy $H$-spaces, then the different ways to multiply and map $n$ points of $X$ are naturally represented by a painted tree, as follows. Nodes not painted correspond to multiplications in $X$, painted nodes correspond to multiplications in $Y$, and the beginning of the painting (along the edges) indicates the moment $f$ is applied to a given point in $X$. (Weak associativity of $X$ and $Y$ justifies the use of planar binary trees, which represent the distinct associations on a set of inputs.) See Figure 2 . 


$$
f(a) *(f(b \bullet c) * f(d)) \longleftrightarrow Y
$$

Figure 2. $A_{n}$-maps between $H$-spaces $(X, \bullet) \stackrel{f}{\longrightarrow}(Y, *)$ are painted trees.

Figure 4 shows two versions of the three-dimensional multiplihedron as Hasse diagrams.

Bi-leveled trees having $n+1$ internal nodes are in bijection with painted trees having $n$ internal nodes, the bijection being given by pruning: Remove the leftmost branch (and hence, node) from a bi-leveled tree to get a tree whose order ideal is the order ideal of the bi-leveled tree, minus the leftmost node. We refer to this as $b i j_{2}$. This mapping and its inverse are illustrated in Figure 3 . The composition of $b j_{2}$ with $\beta$ is just called $\beta$. (We will often use these bijections as identities.)

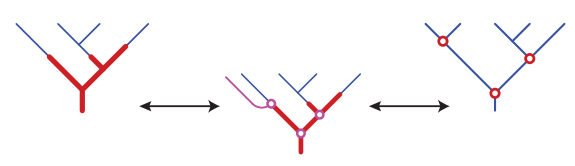

FiguRE 3. Painted trees correspond to bi-leveled trees.
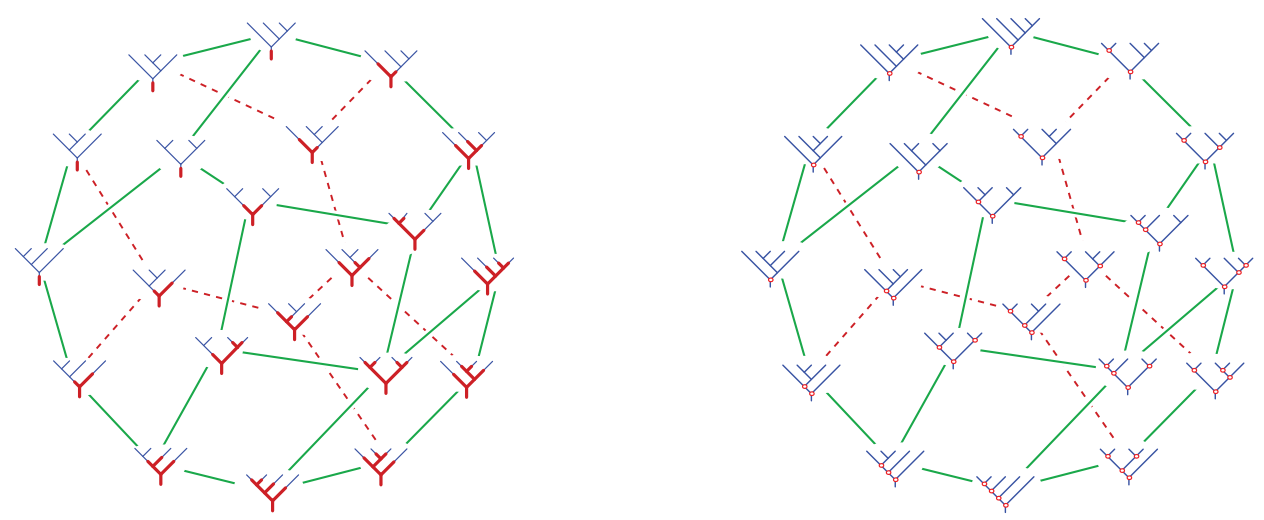

Figure 4. Two Hasse diagrams of the multiplihedra lattice $\mathcal{M}_{4}$, labeled with painted and bi-leveled trees.

Remark 2.4. If the leaves of a painted binary tree are labeled by the elements of a set, it is recognizable as a structure in a certain combinatorial species: the self-composition $\mathcal{Y} \circ \mathcal{Y}$ of binary trees. The structure types of this species are the (unlabeled) binary painted trees themselves. Forgetting the painting in any painted tree is precisely the composition in the operad of binary trees.

Forgetting the levels in a bi-leveled tree (removing the circles) gives a different (from the one just remarked on) projection to binary trees. We denote this by 
$\phi: \mathcal{M}_{n} \rightarrow \mathcal{Y}_{n}$ as in 9]. Now the composition $\phi \circ \beta$ gives the Tonks projection from $\mathfrak{S}_{n}$ to $F y_{n}$, denoted respectively by $\Theta$ in [20], by $\tau$ in [9] and by $\Psi$ in [13].

In [13] Loday and Ronco define a poset map from $\mathcal{Y}_{n}$ to $\mathcal{Q}_{n}$. (They call it $\phi$, here we denote it $\hat{\phi}$ to avoid duplicate naming.) This map takes a tree and gives a vertex of the hypercube $[-1,1]^{n}$ by assigning either +1 or -1 to each of the branches not on a limb. Each branch is assigned its slope, where the tree is drawn with 45 degree angles. Further, they use a bijection (we call it $b i j_{4}$ ) from these vertices to elements of the boolean lattice $\mathcal{Q}_{n}$ defined by including the elements $i \in[n-1]$ which correspond to the coordinates $x_{i}=-1$. The composition of $\hat{\phi}, \phi$ and $\beta$ gives the descents of the permutation.
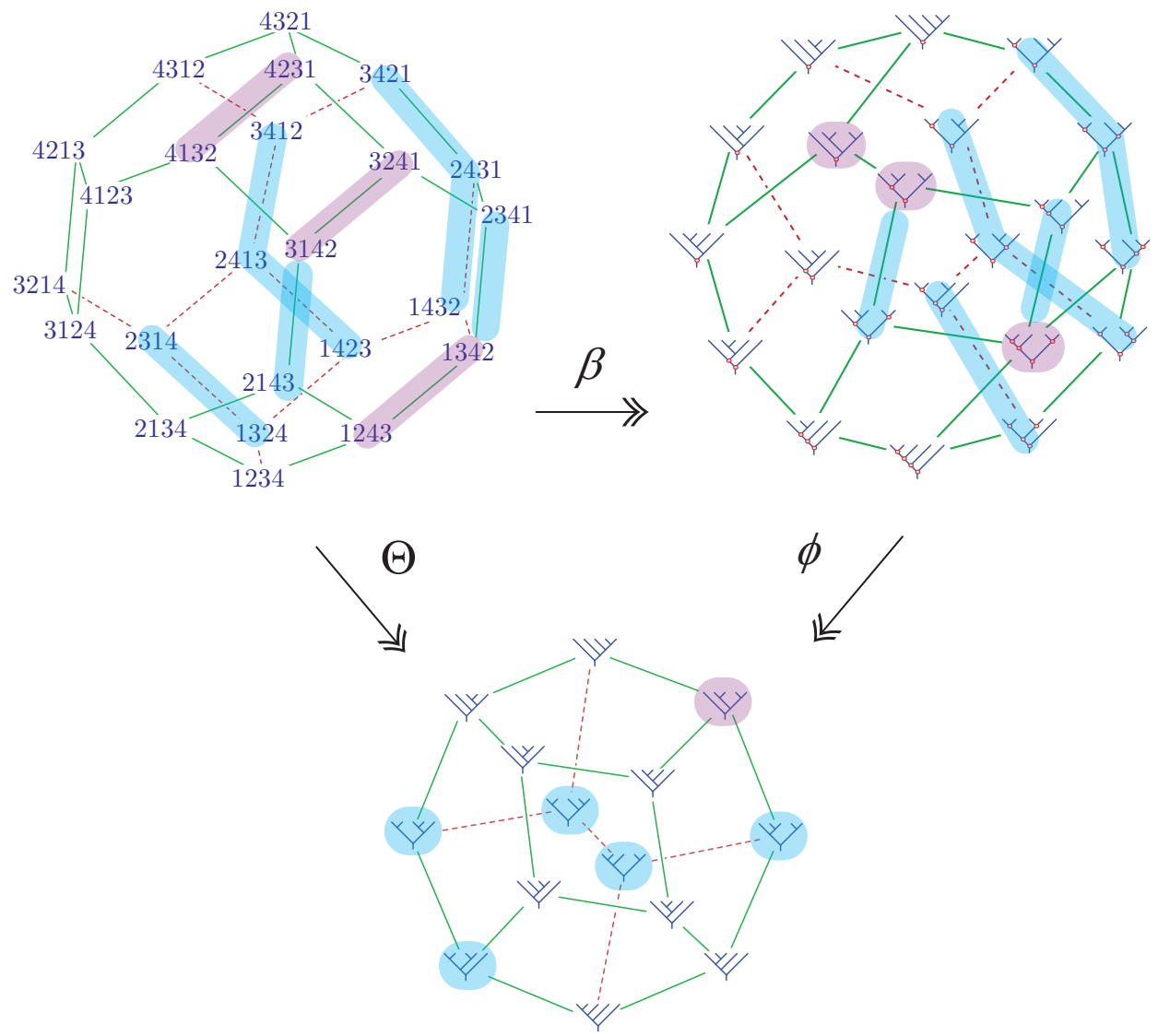

Figure 5. The maps $\Theta=\phi \circ \beta$ shown with retracted intervals shaded.

2.2. Trees with corrollas. We will use the term corolla to describe a rooted tree with one interior node and $n+1$ leaves. In a forest of corollas attached to a binary tree, each corolla may be replaced by a positive weight counting the number of leaves in the corolla. (Alternately, as in [10, the corollas may be replaced by 
combs.) These all give weighted trees.

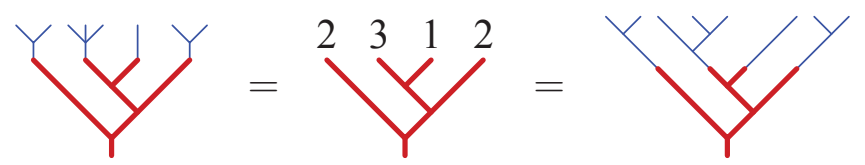

Remark 2.5. By labeling leaves of a comb by the elements of a set, we define a species called $\mathfrak{C}$, which is in fact isomorphic to the species of lists. Labeled weighted trees (as combs grafted to a tree) are recognizable as the structures in the species composition $\mathcal{Y} \circ \mathfrak{C}$.

Let $\mathcal{C K}_{n}$ denote the weighted trees with weights summing to $n+2$. These index the vertices of the $n$-dimensional composihedron, $\mathcal{C K}(n+1)$ [8. This sequence of polytopes parameterizes homotopy maps from strictly associative $H$-spaces to $A_{\infty^{-}}$ spaces.

If we use right combs instead of corollas as the weights on our weighted trees, then the same relations as in $\mathcal{M}_{n}$ give the weighted trees a lattice structure. The joins and meets are found as for painted trees, with the final step of combing the unpainted subtrees. Figure 6 gives two pictures of the composihedron $\mathcal{C K}_{4}$. The 2
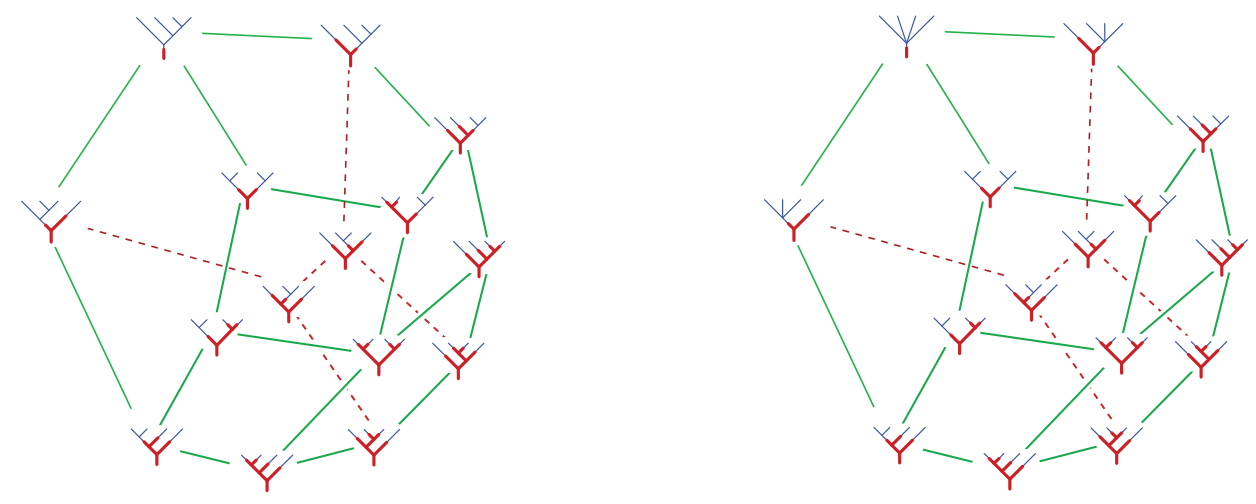

Figure 6. The one-skeleton of the three-dimensional composihedron, as a Hasse diagram labeled by two representations of weighted trees.

and 3-dimensional composihedra $\mathcal{C K}(n)$ also appear as the commuting diagrams in enriched bicategories [8]. As a special case of enriched bicategories, these diagrams appear in the definition of pseudomonoids [1, Appendix C].

On the other hand, attaching a forest of binary trees to a single corolla is really just a way of picturing an ordered forest of binary trees, listed left to right. There is a well known bijection from the set of ordered forests with $n+1$ total leaves to $\mathcal{Y}_{n}$. We call this bijection $b i j_{3}$. It is described by taking the $k$ trees of the forest in left-to-right order and attaching them to a single limb, which will be the new left limb. Thus we can recognize this set as another version of $\mathcal{Y}_{n}$.

Finally we consider trees with $n$ interior nodes obtained by grafting a forest of combs to the leaves of a comb (which is painted). Analogous to 1 , these are weighted combs (or corollas). As these are in bijection with number-theoretic compositions 
of $n+1$, we refer to them as composition trees.

$$
Y Y Y=Y Y Y=3214=(3,2,1,4) \text {. }
$$

Remark 2.6. Leaf-labeled composition trees (where we are labeling the leaves of the forest of combs grafted to a comb) are recognizable as the structures in the species composition $\mathfrak{C} \circ \mathfrak{C}$.

In the next section we will describe maps from the multiplihedra to the hypercubes, but first we note that we will use a different bijection from the set of composition trees to $\mathcal{Q}_{n}$. A composition tree is associated by bijection $b_{i j}$ with the set of vertices that are unpainted.

\section{INTERVAL RETRACTS}

In [9] it is shown that there exists a section of the projection $\beta: \mathfrak{S}_{n} \rightarrow \mathcal{M}_{n}$ which demonstrates $\beta$ to be an interval retract. We review that definition here. Recall that an interval $[a, b]$ of a poset $\mathrm{P}$ is a sub-poset given by $\{x \mid a \leq x \leq b \in \mathrm{P}$. $\}$

A surjective poset map $f: P \rightarrow Q$ from a finite lattice $P$ is an interval retract if the fibers of $f$ are intervals and if $f$ admits an order-preserving section $g: Q \rightarrow P$ with $f \circ g=\mathrm{id}$. Also in [9] there is proven a useful relation between the Möbius functions of $\mathfrak{S}$. and $\mathcal{M}$., which is in fact established there in a general form.

Theorem 3.1. 9] Let the poset map $f: P \rightarrow Q$ be an interval retract, then the Möbius functions $\mu_{P}$ and $\mu_{Q}$ of $P$ and $Q$ are related by the formula

$$
\mu_{Q}(x, y)=\sum_{\substack{f(a)=x \\ f(b)=y}} \mu_{P}(a, b) \quad(\forall x, y \in Q) .
$$

The proof in [9] relies on the fact that the intersection of two intervals in a lattice is again an interval.

Here we define eight closely related maps in order to demonstrate four new interval retracts: first four projections, each associated to a corresponding section.
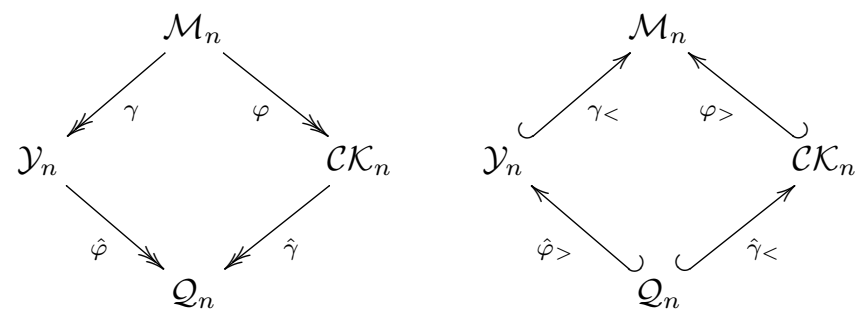

The maps $\gamma$ and $\hat{\gamma}$ operate by replacing the painted portion with a corolla, while $\varphi$ and $\hat{\varphi}$ replace the unpainted forest with a forest of corollas.

We define the sections $\gamma_{<}$and $\hat{\gamma}_{<}$by replacing painted corollas with left combs, while $\varphi_{>}$and $\hat{\varphi}_{>}$are defined by replacing unpainted corollas with right combs

The main result will be that each paired projection and section between the same two lattices together define an interval retract. In figure 7 we demonstrate all the projections and bijections described above.

Theorem 3.2. The map $\gamma$ is an interval retract from $\mathcal{M}_{n}$ to $\mathcal{Y}_{n}$. 


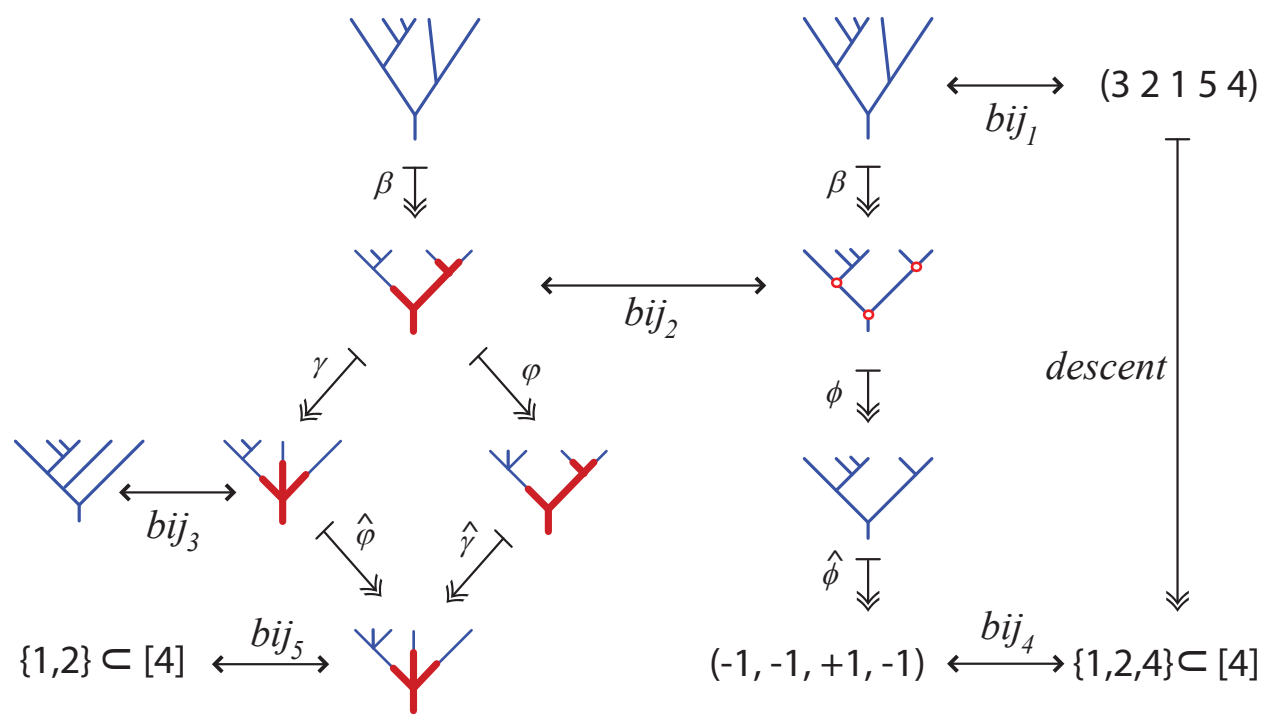

FiguRe 7. Projections and bijections in this paper.

Proof. We use the section $\gamma_{<}$. Thus we need to show four things: that $\gamma^{-1}(t)$ is an interval in $\mathcal{M}_{n}$ for any $t \in \mathcal{Y}_{n}$; that $\gamma$ and $\gamma_{<}$both preserve order, and that $\gamma \circ \gamma_{<}$is the identity map. This last fact is straightforward, since it constitutes first removing and then replacing an unpainted forest on its painted comb.

Consider $t \in \mathcal{Y}_{n}$ with the $k$-forest $f$ of subtrees with initial nodes on the left limb of $t$. To show that inverse images of $\gamma$ are intervals, we point out that $\gamma^{-1}(t)$ is the set of painted trees with unpainted forest $f$ and any painted portion. This is an interval since its elements comprise all those between a unique min and max given by minimizing and maximizing the painted portion. In fact, the interval is isomorphic to a copy of the Tamari lattice $\mathcal{Y}_{k-1}$.

Next to show that $\gamma$ preserves the order, we let $a<b \in \mathcal{M}_{n}$. This means that $t_{a} \leq t_{b}$ in the Tamari order, and that $P_{a} \supseteq P_{b}$. Now we may visualize the action of $\gamma$ as a series of smaller steps: first we make all possible Tamari moves in the painted region of $a$ that each yield sequentially lesser trees. Then we attach a new branch to the left-most painted point of $a$, and finally forget the painting altogether. The same basic steps are performed to find $\gamma(b)$. Since $P_{b} \subseteq P_{a}$ we can see the relation $\gamma(b) \geq \gamma(a)$ by the series of Tamari moves to get from $b$ to $a$ followed by more moves resulting from the possibly additional painted nodes of $a$.

To show that if $q<s \in \mathcal{Y}_{n}$ implies that $\gamma_{<}(q) \leq \gamma_{<}(s)$, we consider the string of Tamari covering moves that relate $q$ to $s$. Recall that $\gamma<$ takes the $k$-forest $f$ of sub-trees attached along the left limb of $q$ and instead attaches them to a minimal painted $k$-tree, that is, they are grafted to a painted left comb with $k$ branches. Alternately this is described by simply pruning away the leftmost leaf of $q$ and painting the nodes of $q$ along the leftmost branch. We see that $t_{\gamma_{<}(q)} \leq t_{\gamma_{<}(s)}$ by noting that the moves between them are the same as those from $q$ to $s$. Then we note that we have $P_{\gamma_{<}(q)} \supseteq P_{\gamma_{<}(s)}$ since any move from $q$ to $s$ either subtracts from 


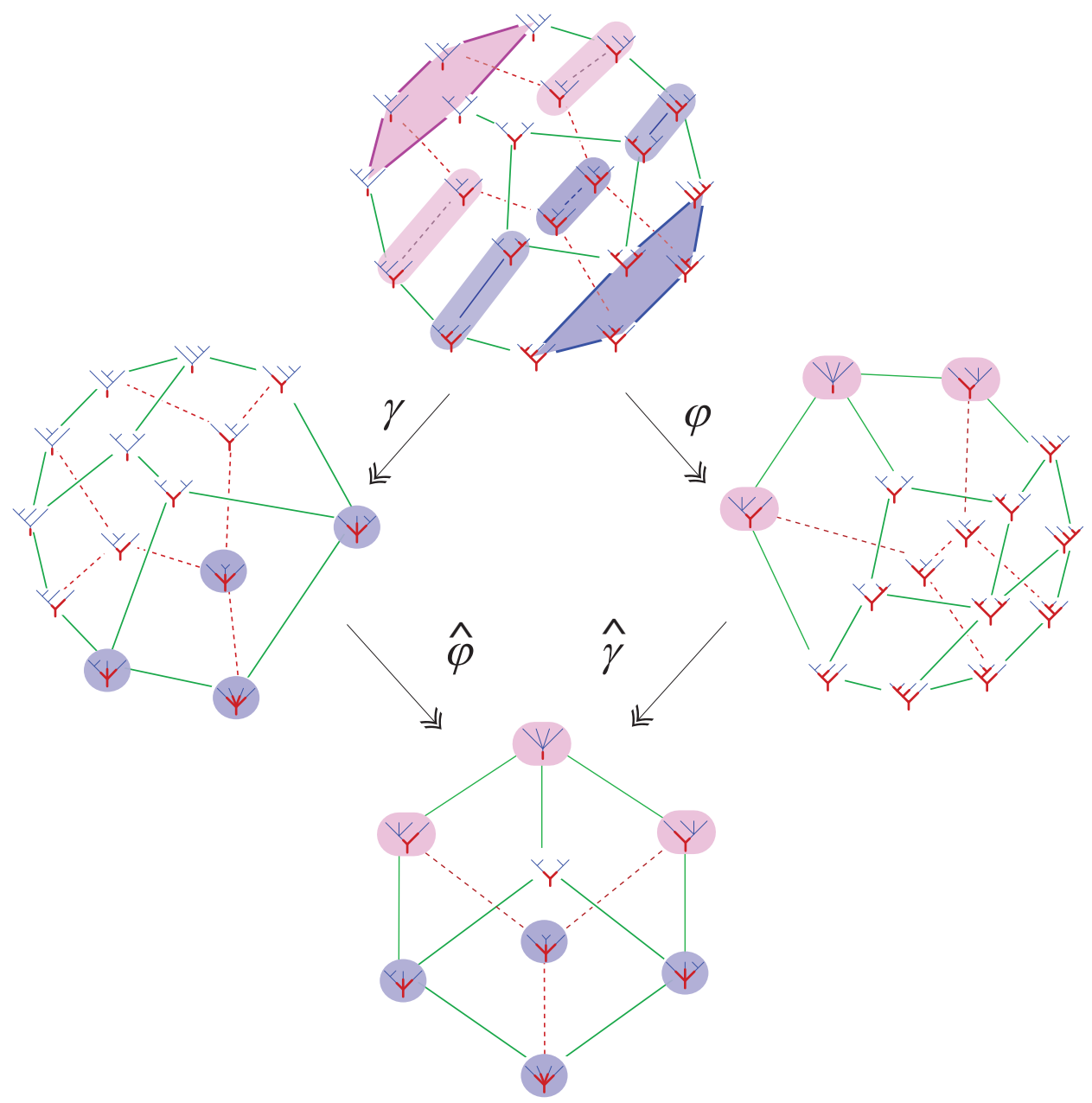

Figure 8. The four projections in dimension 3, with shaded intervals retracted.

the set of painted nodes in the eventual image (if the move involves a node on the leftmost branch of $q$ ) or leaves that set unchanged.

Theorem 3.3. The map $\varphi$ is an interval retract from $\mathcal{M}_{n}$ to $\mathcal{C K}_{n}$.

Proof. We use the section $\varphi_{>}$. Again we need to show four things: that $\varphi^{-1}(t)$ is an interval in $\mathcal{M}_{n}$ for any $w \in \mathcal{C K}_{n}$; that $\varphi$ and $\varphi>$ both preserve order, and that $\varphi \circ \varphi_{>}$is the identity map. This last fact is straightforward, since both maps will be the identity in this case $-\varphi_{>}$will always be and $\varphi$ will be the identity when applied to a painted tree with a forest of unpainted right combs.

Recall that $\varphi$ involves replacing an unpainted forest $f$ with a forest of right combs. (Sometimes alternately drawn as corollas or just a number.) Thus the fiber $\varphi^{-1}(w)$ for $w \in \mathcal{C K}_{n}$ is a collection of painted trees in $\mathcal{M}_{n}$ which share the same set of painted nodes, and the same binary tree as the subtree made up of those 


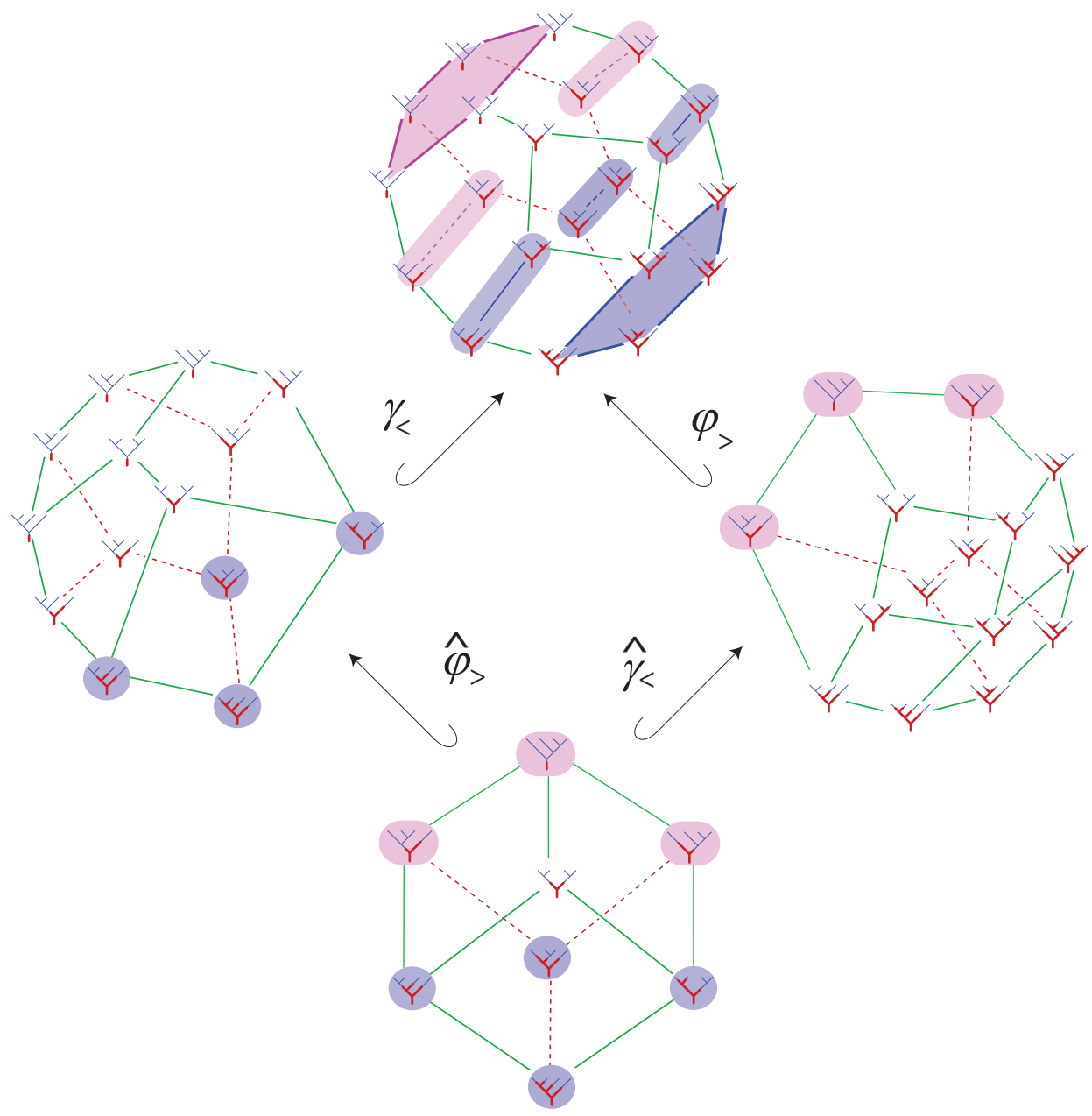

Figure 9. The four sections in dimension 3. Here we label the elements of $\mathcal{Y}_{n}, \mathcal{C} \mathcal{K}_{n}$ and $\mathcal{Q}_{n}$ using left and right combs so that the sections can be seen as inclusions.

painted nodes - but which may have any forest of unpainted trees agreeing with those facts. Thus the fiber is an interval bounded by choosing that forest to be all left or all right combs. In fact this is a cartesian product of associahedra.

Next to show that $\varphi$ preserves the order, we let $a<b \in \mathcal{M}_{n}$. This means that $t_{a} \leq t_{b}$ in the Tamari order, and that $P_{a} \supseteq P_{b}$. First note that if $P_{a}=P_{b}$, then $\varphi(a)<\varphi(b)$ by Tamari moves in the painted nodes. If $P_{a} \supset P_{b}$, consider the forest of unpainted right combs of $\varphi(b)$. Each of these combs has a leftmost node $k$. If the corresponding node $k$ of $\varphi(a)$ is painted, then we can see the relation as first performing Tamari moves on the right comb of $\varphi(b)$ until we have the binary tree supported by node $k$ of $\varphi(a)$, and then allowing the paint level to rise to cover node $k$ and any additional nodes to match $\varphi(a)$. These moves performed on each unpainted comb of $\varphi(b)$ give us the result. 
The section $\varphi_{>}$is very simple; it merely returns us to the maximum of the fiber. In fact, if we are using right combs for our weighted trees then this section is the identity map, and so order is clearly preserved.

Theorem 3.4. The map $\hat{\varphi}$ is an interval retract from $\mathcal{Y}_{n}$ to $\mathcal{Q}_{n}$.

Proof. It is easiest to see this when viewing $\mathcal{Y}_{n}$ in its incarnation as ordered forests of binary trees, grafted onto painted left combs. Then the ordering of $\mathcal{Y}_{n}$ is directly inherited from $\mathcal{M}_{n}$, and the map $\hat{\varphi}$ is the same as $\varphi$. Thus the facts that the fibers are intervals and that $\hat{\varphi} \circ \hat{\varphi}_{>}$is the identity are already proven.

Now the elements of $\mathcal{Q}_{n}$ are being drawn as composite trees (using combs or corollas) but we need to check that the usual ordering by inclusion of subsets (of unpainted nodes) agrees with the tree order. That is, if $p<q$ as elements of $\mathcal{Y}_{n}$ (each drawn as an unpainted forest grafted to a left comb) then $\hat{\varphi}(p) \leq \hat{\varphi}(q)$. By viewing two elements of $\mathcal{Q}_{n}$ as forests of right combs grafted to painted left combs, we see that the only relation inherited from $\mathcal{M}_{n}$ is that of the containment of the sets of painted nodes. Thus since in $\mathcal{M}_{n}$ a larger set of painted nodes is a lesser element, here a smaller set of unpainted nodes is the lesser element.

Finally the section $\hat{\varphi}_{>}$is given by inclusion of the composite tree as a forest of right combs grafted to a painted left comb, which ensures that the ordering is preserved.

Theorem 3.5. The map $\hat{\gamma}$ is an interval retract from $\mathcal{C} \mathcal{K}_{n}$ to $\mathcal{Q}_{n}$.

Proof. We already view an element of $\mathcal{C K}_{n}$ as a forest of right combs grafted to a painted binary tree. Viewing $\hat{\gamma}$ as replacing the painted nodes with a left comb, we see the proof proceeds just as for $\hat{\varphi}$.

\section{LATTICES AND POLYTOPES.}

Next we point out that the four interval retracts just defined extend to well known cellular projections of the polytopes. These projections are not the same ones that appear in the work of Reading [14], Loday and Ronco [13], or Tonks [20]. Rather they are found implicitly in the work of Boardman and Vogt on maps of homotopy $H$-spaces [4].

Recall that the combinatorial lattices of trees we have discussed here all occur conveniently as the labels of vertices on convex polytopes. The Hasse diagrams are specific drawings of the 1-skeleton of each polytope. The polytopes are associated to another, entirely different, lattice: the poset of their faces, with the empty set adjoined as least element. It is an open question as far as I know whether there is any describable relationship between the two lattices, for instance between the Tamari lattice and the face-poset of the associahedron.

As indicated in the introduction (by our use of the same symbol for both polytope and lattice) we have the following correspondence: binary trees label vertices of the associahedra; ordered binary trees the permutohedra; painted binary trees the multiplihedra, weighted trees the composihedra; composition trees the hypercubes. The higher dimensional faces of these polytopes are all associated to further generalizations of the trees in question, by allowing more non-binary nodes and by allowing painting to end precisely on a node.

The Hopf algebras of binary trees, ordered trees and Boolean subsets have all been extended to larger Hopf algebras on the faces of the corresponding polytopes. 
This was achieved by Chapoton in [6]. It is the topic of future study that similar expansions exist for the multiplihedra and composihedra.

Here we show how the projections discussed in this paper appear as collapsing of the faces of the polytopes whose vertices they act upon. Figure 10 shows an alternate "above" view of the permutohedron. This is given in order to facilitate contrasting the various projection maps.

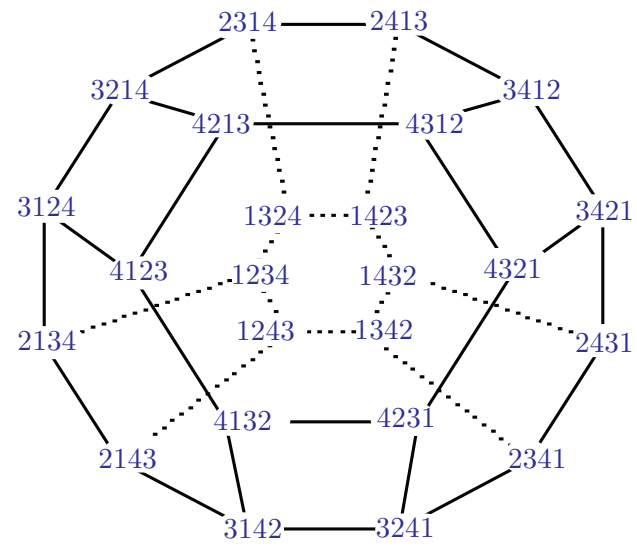

FiguRE 10. The 3d permutohedron, alternate view.

Figure 11 offers contrast and comparison of our new maps to the classic projections, showing the faces that are retracted. We use the "above" view of the posets. In the pictured $3 \mathrm{~d}$ case it is apparent that the two compositions of maps, $\hat{\phi} \circ \phi \circ \beta$ (which is the Tonks projection) and $\hat{\varphi} \circ \gamma \circ \beta$, have quite different actions on the permutohedron. The number of collapsed cells are the same in both composite projections, but in the first the image of the collapsed cells (4 hexagons and 4 rectangles) is a copy of $S^{1}$ where in the second the image (of 2 hexagons and 6 rectangles) consists of two disjoint star graphs.

Next, for comparison, is the Tonks projection again, factored through the cyclohedron as in [11]. Finally for further contrast we include the projection $\eta$ defined by Reading in his theory of Cambrian lattices [15].

The factorization of the Tonks projection, $\Theta=\theta_{2} \circ \theta_{1}$, through the cyclohedron seen in Figure 11 deserves some special mention. First, this factorization is defined in greater generality [11] in terms of tubings of simple graphs.

Definition 4.1. Let $G$ be a finite connected simple graph, with $n$ numbered nodes. A tube is a set of nodes of $G$ whose induced graph is a connected subgraph of $G$. Two tubes $u$ and $v$ may interact on the graph as follows:

(1) Tubes are nested if $u \subset v$.

(2) Tubes are far apart if $u \cup v$ is not a tube in $G$, that is, the induced subgraph of the union is not connected, or none of the nodes of $u$ are adjacent to a node of $v$.

Tubes are compatible if they are either nested or far apart. We call $G$ itself the universal tube. A tubing $T$ of $G$ is a set of tubes of $G$ such that every pair of tubes in $T$ is compatible; moreover, we force every tubing of $G$ to contain (by default) its 
a
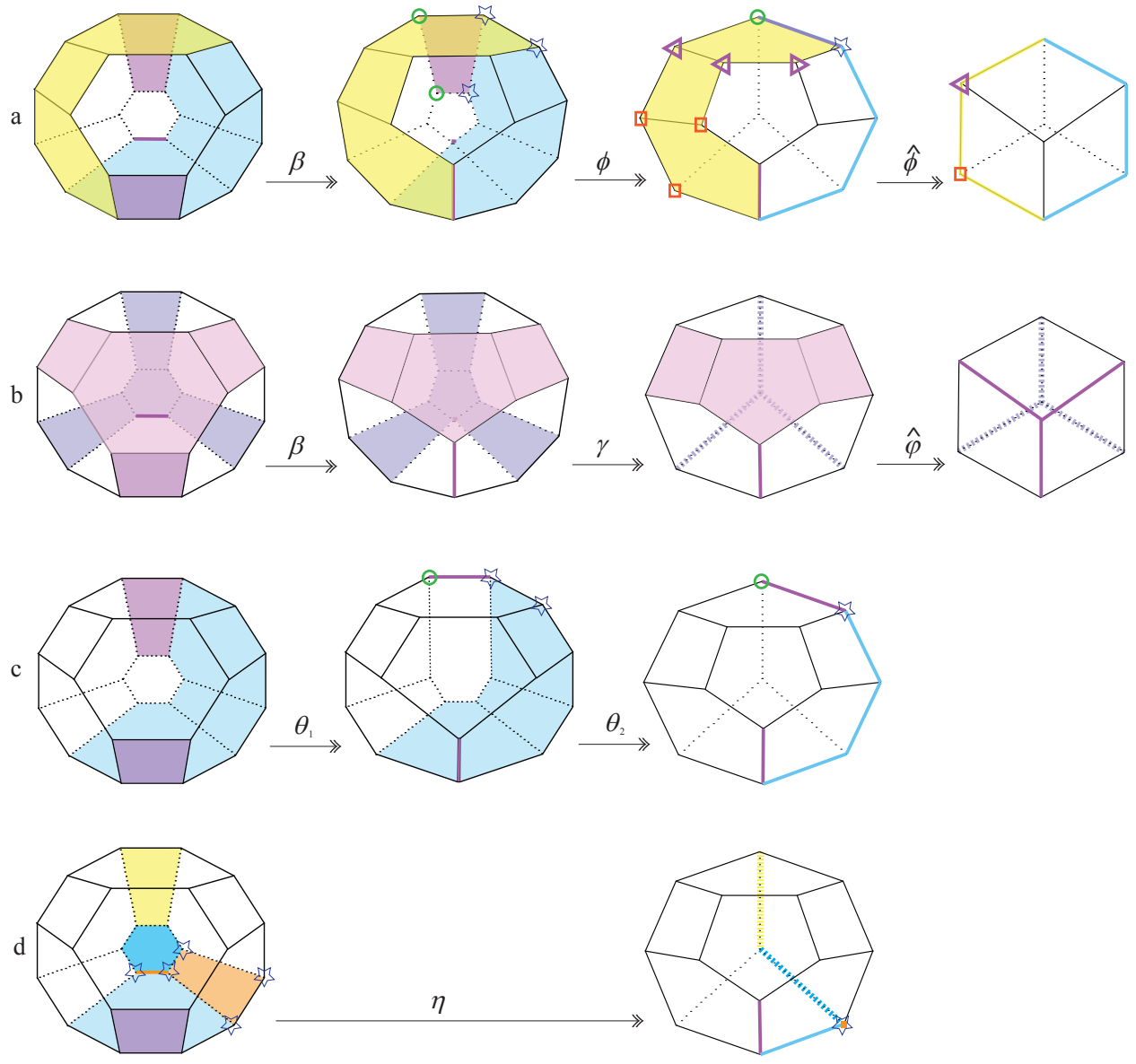

Figure 11. In a, b, c and d the permutohedron is oriented as in Figure 10. The shaded facets and edges are collapsed in succession to similarly shaded edges and points. In a the circled and starred vertices in the domain of $\phi$ are mapped one and all to the circled (respectively starred) vertex in the range, and likewise for the squared and triangled vertices in the domain and range of $\hat{\phi}$. In $\mathrm{b}$ both $\gamma$ and $\hat{\varphi}$ collapse a pentagon in their respective domains to a single vertex in their respective ranges. In $\mathrm{c}$, the central polytope is the $3 \mathrm{~d}$ cyclohedron. Finally in $\mathrm{d} \eta$ takes all the starred vertices to a single vertex.

universal tube. By the term $k$-tubing we refer to a tubing made up of $k$ tubes, for $k \in\{1, \ldots, n\}$.

Theorem 4.2. [5, Section 3] For a graph $G$ with $n$ nodes, the graph associahedron $\mathcal{K} G$ is a simple, convex polytope of dimension $n-1$ whose face poset is isomorphic to the set of tubings of $G$, ordered such that $T \prec T^{\prime}$ if $T$ is obtained from $T^{\prime}$ by adding tubes. 
The vertices of the graph associahedron are the $n$-tubings of $G$. Faces of dimension $k$ are indexed by $(n-k)$-tubings of $G$.

As seen in [5], the permutohedron $\mathfrak{S}_{n}=\mathcal{K} G$ where $G$ is the complete graph on $n$ nodes; the associahedron $\mathcal{Y}_{n}=\mathcal{K} G$ where $G$ is the path graph on $n$ nodes; the cyclohedron is $\mathcal{W}_{n}=\mathcal{K} G$ when $G$ is the cycle on $n$ nodes; and the stellohedron is $\mathcal{K} G$ when $G$ is the star graph on $n$ nodes.

The question might be asked: how easily may the weak order on permutations and the Tamari order be generalized to $n$-tubings on a graph with nodes numbered $1, \ldots, n$ ? In order to describe the ordering we give the covering relations. We can use the same notation as when comparing tubings in the poset of faces of the graph associahedron since in that poset the $n$-tubings are not comparable.

Definition 4.3. Two $n$-tubings $T, T^{\prime}$ are in a covering relation $T^{\prime} \prec T$ if they have all the same tubes except for one differing pair. We actually compare the outermost nodes, one from each of the pair of differing tubes. The outermost node of a tube is the node that is included in no other smaller sub-tube of the tubing. If the number of that node is greater for $T$, then $T$ covers $T^{\prime}$.

Note that each such covering relation corresponds to a unique $(n-1)$-tubing: the one resulting from removing the differing tubes. Thus the covering relations correspond to the edges of the graph associahedron.

For example, in Figure 12 we show a covering relation between two tubings on the complete graph on four numbered nodes. This figure also demonstrates the bijection between $n$-tubings and permutations of $[n]$. The nodes are the inputs for the permutation, and the output is the relative tube size. E.g., in the left-hand permutation the image of 2 is 1 , and so we put the smallest tube around 2. To see the relation via tubes, we write down the sets of nodes in each tube. Only one pair of tubes differs. We compare the two numbered nodes of these which are in no smaller tubes. Here $(3124) \prec(4123)$ since $1<4$.

(3 112 2 4 )

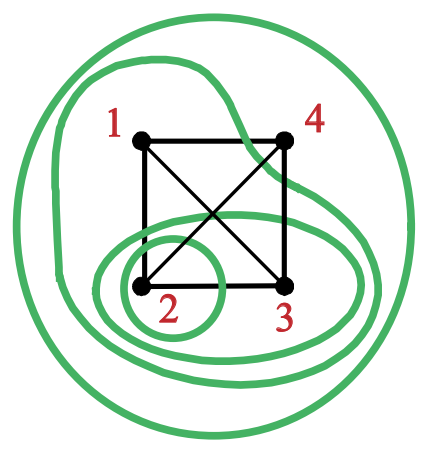

$\{2\}$

$\{3,2\}$

$\{3,2,1\}$ $\left(\begin{array}{llll}4 & 1 & 2 & 3\end{array}\right)$

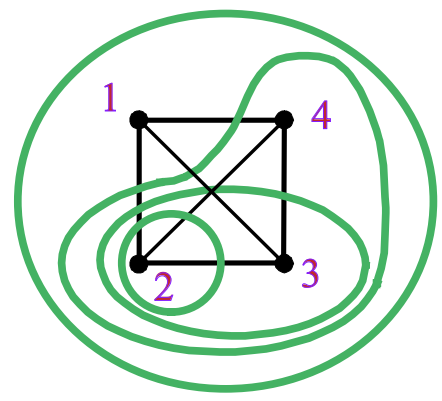

$\{2\}$

$\{3,2\}$

$\{4,3,2\}$

FIgURE 12. A covering relation in the weak order on permutations. 
It turns out that the relation generated by these covering relations of tubings has been independently demonstrated to be a poset by Ronco [16]. In her article, the poset we have described on $n$-tubings of a graph is seen as the restriction of a larger poset on all the tubings of a graph.

Figure 13 shows the lattice that results from the cycle graph, rocovering the cyclohedron in dimension 3. The Hasse diagram is combinatorially equivalent to the 1-skeleton of the cyclohedron. Notice that this is quite different from the type $B_{3}$ Cambrian lattice described by Reading in this volume [15, despite the fact that the latter also is combinatorially equivalent to the 1-skeleton of the cyclohedron. Figure 14 shows the corresponding lattice on 4-tubings of the star graph on 4 nodes. This Hasse diagram is combinatorially equivalent to the 1-skeleton of the 3d stellohedron. Figure 15 shows both the cyclohedron and stellohedron lattices again, unlabeled, with a different view of each polytope for comparison.

We note that as seen in Ronco's article [16], the Tamari lattice is found as the lattice of $n$-tubings on the path graph with nodes numbered $1, \ldots, n$ in the order that they are connected by edges. Several open questions present themselves: for one, we notice that the 3-dimensional graph associahedra pictured here have associated posets which upon inspection prove to be lattices-it is not clear that they always are.

\section{Algebraic implications of interval Retracts}

Finally we point out the importance of these lattices to the Hopf algebras defined as spans of their elements. For each of the lattices studied here, there is a graded vector space given by the direct product of the spans of the vertices of the $n$ dimensional polytope. For instance a vector space of binary trees is defined as:

$$
\mathcal{Y} \text { Sym }=\bigoplus_{n \geq 0} \operatorname{span} \mathcal{Y}_{n}
$$

The binary trees index a basis, called the fundamental basis and denoted $\left\{F_{t} \mid t \in\right.$ $\left.\mathcal{Y}_{n}\right\}$. There is a graded Hopf algebra structure on this vector space, well studied in [3. Similarly

$$
\mathfrak{S} S y m=\bigoplus_{n \geq 0} \operatorname{span} \mathfrak{S}_{n}
$$

is a graded Hopf algebra on ordered trees, well studied in [2].

Here we will restrict our attention to the coalgebra structures, which interact in important ways with the lattice structures. The remainder of this section is taken in part from [10]. It is included in order to demonstrate the algebraic importance of the lattice structures.

5.1. Coalgebras of trees. We define splitting a binary tree $w$ along the path from a leaf to the root to yield a pair of binary trees,

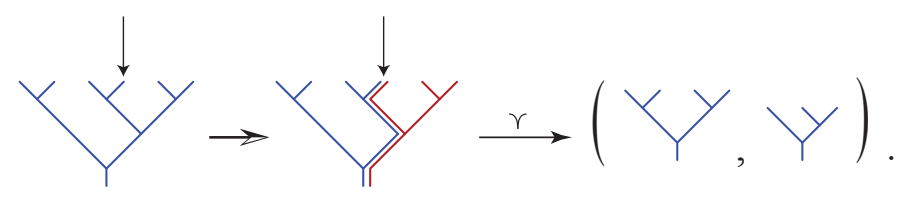

Write $w \stackrel{\curlyvee}{\rightarrow}\left(w_{0}, w_{1}\right)$ when the pair of trees $\left(w_{0}, w_{1}\right)$ is obtained by splitting $w$. 


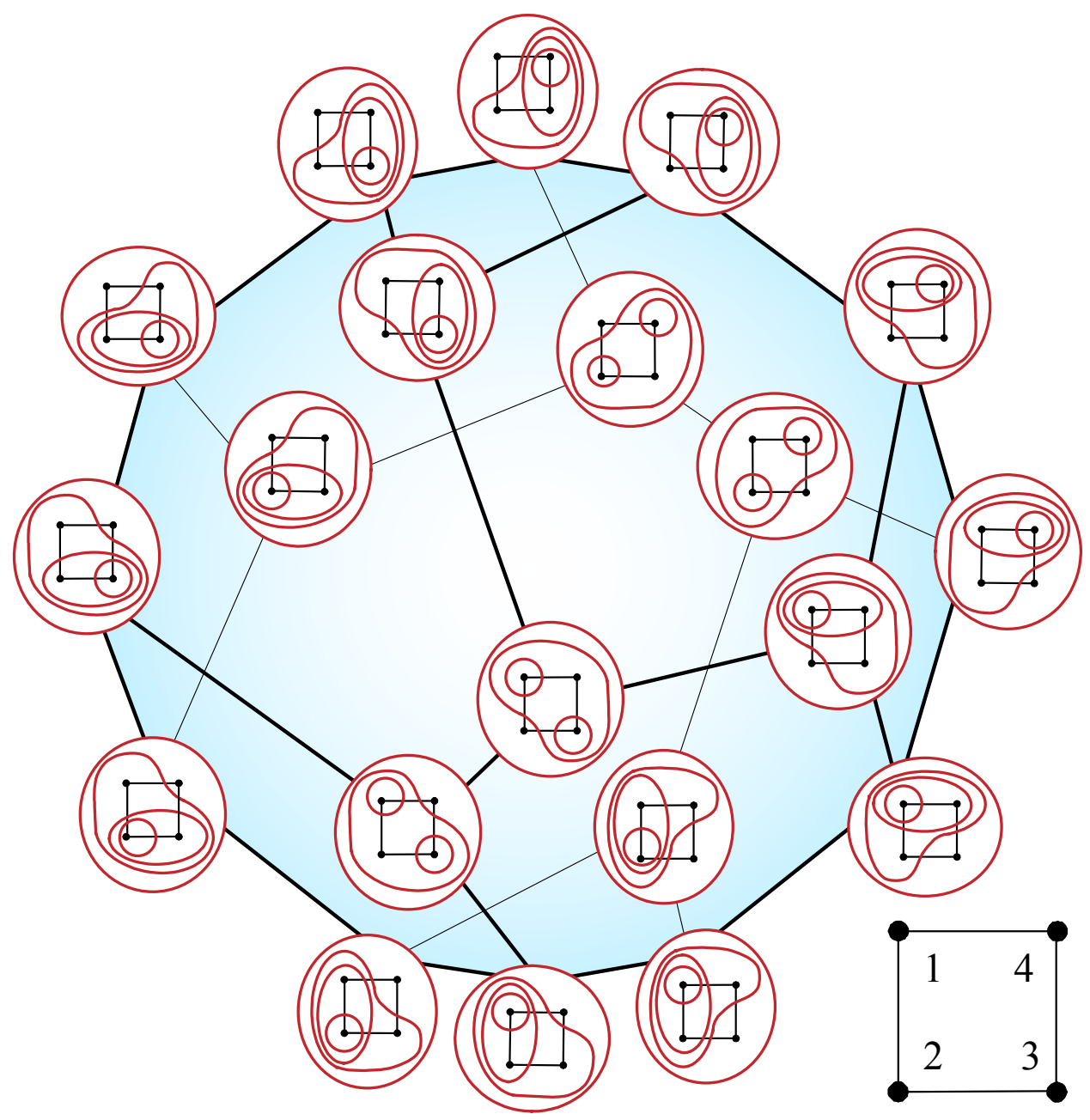

Figure 13. This Hasse diagram is labeled by tubings of the cycle graph, with nodes numbered $1-4$. The covering relations are also a picture of the edges of the cyclohedron.

Definition 5.1 (Coproduct on $\mathcal{Y}$ Sym). Given a binary tree $t$, define the coproduct in the fundamental basis by

$$
\Delta\left(F_{t}\right)=\sum_{\substack{r \\ t \rightarrow\left(t_{0}, t_{1}\right)}} F_{t_{0}} \otimes F_{t_{1}} .
$$

Here is an example:

$$
\Delta(F Y)=1 \otimes F_{Y}+F_{Y} \otimes F_{Y}+F_{Y} \otimes 1
$$

5.2. Cofree composition of coalgebras. The following is excerpted with edits from [10. Let $\mathcal{C}$ and $\mathcal{D}$ be graded coalgebras. We form a new coalgebra $\mathcal{E}=\mathcal{D} \circ \mathcal{C}$ 


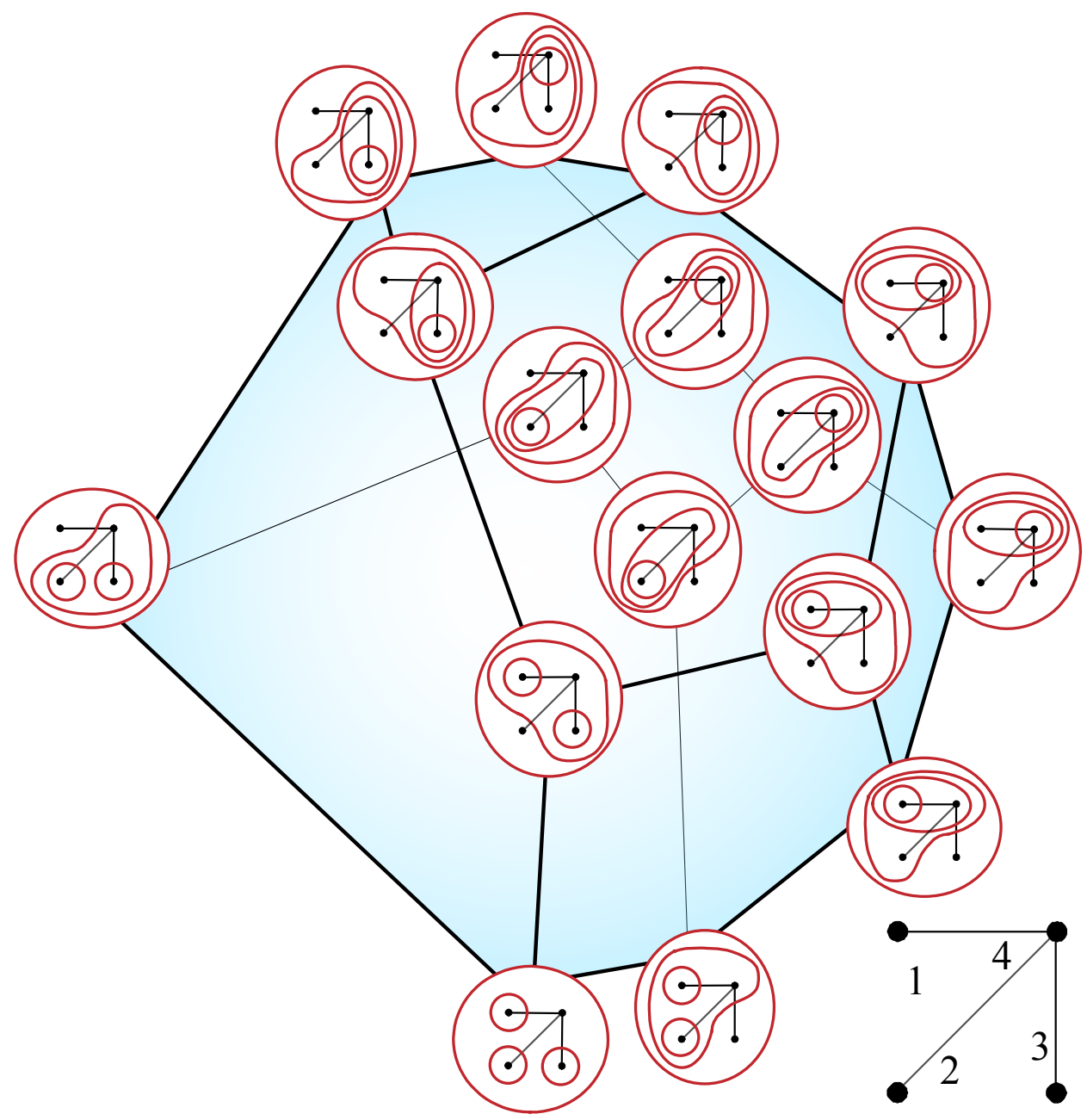

Figure 14. This Hasse diagram is labeled by tubings of the star graph, with nodes numbered $1-4$. The covering relations are also a picture of the edges of the stellohedron.

on the vector space

$$
\mathcal{D} \circ \mathcal{C}:=\bigoplus_{n \geq 0} \mathcal{D}_{n} \otimes \mathcal{C}^{\otimes(n+1)}
$$

We write $\mathcal{E}=\bigoplus_{n \geq 0} \mathcal{E}_{(n)}$, where $\mathcal{E}_{(n)}=\mathcal{D}_{n} \otimes \mathcal{C}^{\otimes(n+1)}$. This gives a coarse coalgebra grading of $\mathcal{E}$ by $\mathcal{D}$-degree. There is a finer grading of $\mathcal{E}$ by total degree, in which a decomposable tensor $c_{0} \otimes \cdots \otimes c_{n} \otimes d$ (with $d \in \mathcal{D}_{n}$ ) has total degree $\left|c_{0}\right|+\cdots+$ $\left|c_{n}\right|+|d|$. Write $\mathcal{E}_{n}$ for the linear span of elements of total degree $n$.

Example 5.2. This composition is motivated by a grafting construction on trees. Let $d \times\left(c_{0}, \ldots, c_{n}\right) \in \mathcal{Y}_{n} \times\left(\mathcal{Y}_{\cdot^{n+1}}\right)$. Define $\circ$ by attaching the forest $\left(c_{0}, \ldots, c_{n}\right)$ to 

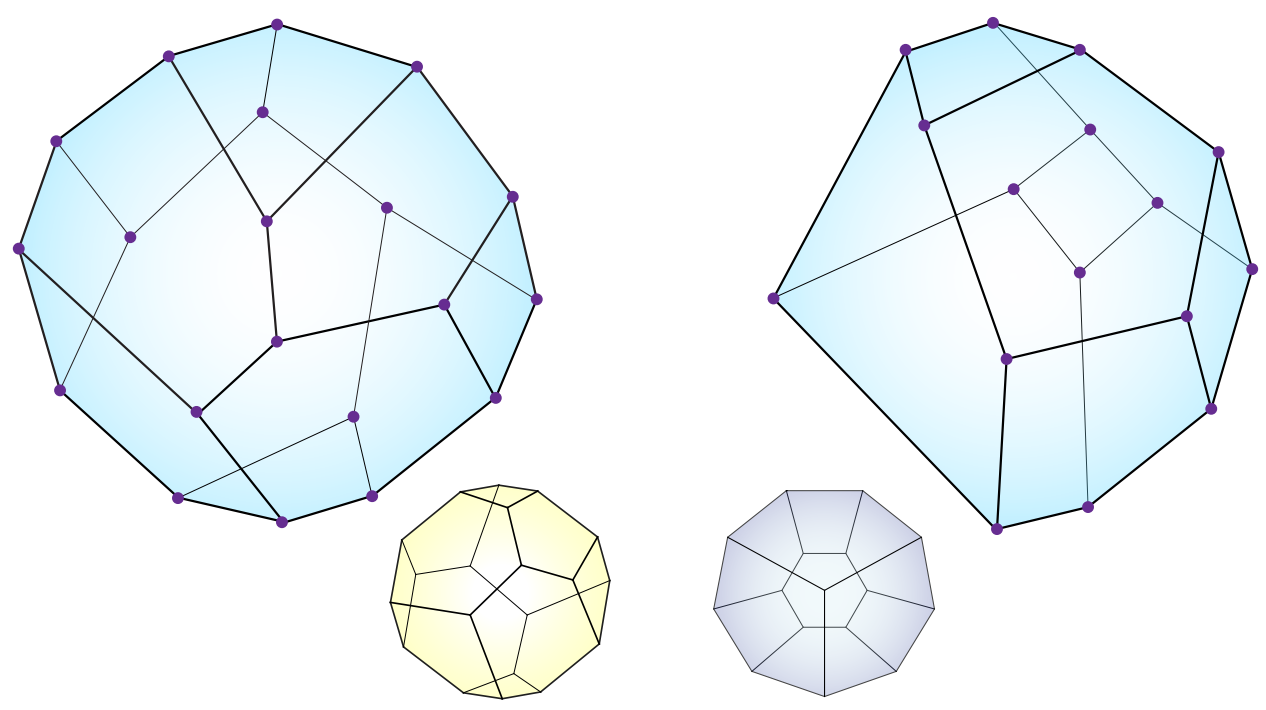

Figure 15. On the left is the Hasse diagram for $n$-tubings of the cycle graph, with the cyclohedron pictured below for comparison. On the right is the Hasse diagram for $n$-tubings of the star graph, with the stellohedron pictured below.

the leaves of $d$ while remembering $d$,

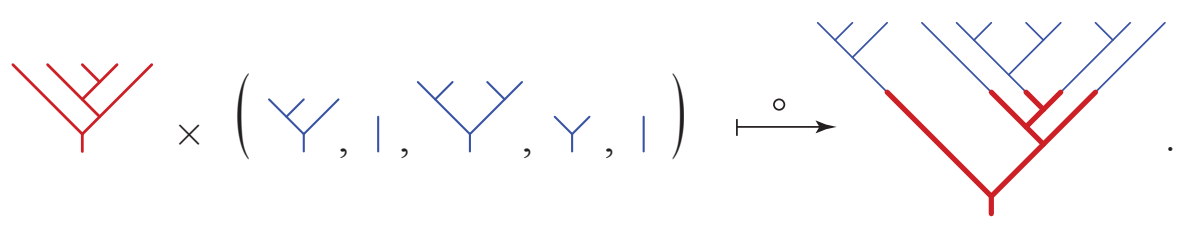

This is precisely the type of tree called a painted tree in Section 2. Applying this construction to the indices of basis elements of $\mathcal{C}$ and $\mathcal{D}$ and extending by multilinearity gives $\mathcal{C} \circ \mathcal{D}$.

Motivated by this example, we represent an decomposable tensor in $\mathcal{D} \circ \mathcal{C}$ as

$$
\left(c_{0} \cdots \cdots c_{n}\right) \circ d \quad \text { or } \quad \frac{c_{0} \cdots \cdot c_{n}}{d}
$$

to compactify notation.

5.3. The coalgebra of painted trees. Let $\mathcal{M}_{n}$ be the poset of painted trees on $n$ internal nodes. Then the vector space $\mathcal{P}$ Sym $=\mathcal{Y}$ Sym $\circ \mathcal{Y}$ Sym may be directly given by:

$$
\mathcal{P} \text { Sym }=\bigoplus_{n \geq 0} \operatorname{span} \mathcal{M}_{n}
$$

We reproduce the compositional coproduct defined in Section 2 of [10]. 
Definition 5.3 (Coproduct on $\mathcal{P} S y m$ ). Given a painted tree $p$, define the coproduct in the fundamental basis $\left\{F_{p} \mid p \in \mathcal{M}\right.$. $\}$ by

$$
\Delta\left(F_{p}\right)=\sum_{\substack{\curlyvee \\ p \rightarrow\left(p_{0}, p_{1}\right)}} F_{p_{0}} \otimes F_{p_{1}},
$$

where the painting in $p$ is preserved in the splitting $p \stackrel{\curlyvee}{\rightarrow}\left(p_{0}, p_{1}\right)$.

The counit $\varepsilon$ satisfies $\varepsilon\left(F_{p}\right)=\delta_{0,|p|}$, the Kronecker delta, as usual for graded coalgebras.

5.3.1. Primitives in the coalgebras of trees and painted trees. Now for the discussion of how the lattice structure found by Tamari really impacts the algebraic structure. Recall that a primitive element $x$ of a coalgebra is such that $\Delta x=1 \otimes x+x \otimes 1$. Theorem 2.4 of [10] describes the primitive elements of $\mathcal{P}$ Sym $=\mathcal{Y}$ Sym $\circ \mathcal{Y}$ Sym in terms of the primitive elements of $\mathcal{Y}$ Sym. We recall the description of primitive elements of $\mathcal{Y}$ Sym as given in [3].

Let $\mu$ be the Möbius function of $\mathcal{Y}_{n}$ which is defined by $\mu(t, s)=0$ unless $t \leq s$,

$$
\mu(t, t)=1, \quad \text { and } \quad \mu(t, r)=-\sum_{t \leq s<r} \mu(t, s) .
$$

We define a new basis for $\mathcal{Y}$ Sym using the Möbius function. For $t \in \mathcal{Y}_{n}$, set

$$
M_{t}:=\sum_{t \leq s} \mu(t, s) F_{s}
$$

Then the coproduct for $\mathcal{Y} S y m$ with respect to this $M$-basis is still given by splitting of trees, but only at leaves emanating directly from the right limb above the root:

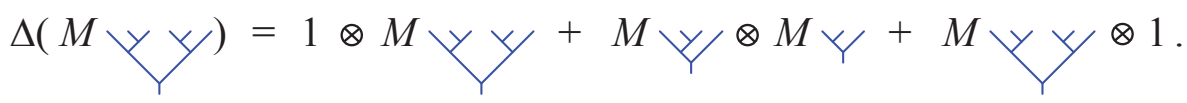

A tree $t \in \mathcal{Y}_{n}$ is progressive if it has no branching along the right branch above the root node. A consequence of the description of the coproduct in this $M$-basis is Corollary 5.3 of [3] that the set $\left\{M_{t} \mid t\right.$ is progressive $\}$ is a linear basis for the space of primitive elements in $\mathcal{Y}$ Sym.

Now according to Theorem 2.4 of $[10]$ the cogenerating primitives in $\mathcal{P}$ Sym are of two types:

$$
\frac{1 \cdot c_{1} \cdots \cdot c_{n-1} \cdot 1}{M_{t}} \quad \text { and } \quad \frac{M_{t}}{1}
$$

where $t$ is a progressive tree. Figure 16 shows examples.

The primitives can be described in terms of Möbius inversion on certain subintervals of the multiplihedra lattice. For primitives of the first type, the subintervals are those with a fixed unpainted forest of the form $(|\cdot t \cdots \cdot s \cdot|)$. For primitives of the second type, the subinterval consists of those trees whose painted part is trivial, i.e. only the root is painted. Each subinterval of the first type is isomorphic to $\mathcal{Y}_{m}$ for some $m \leq n$, and the second subinterval is isomorphic to $\mathcal{Y}_{n}$. Figure 17 shows the multiplihedron lattice for $\mathcal{M}_{4}$, with these subintervals highlighted. 


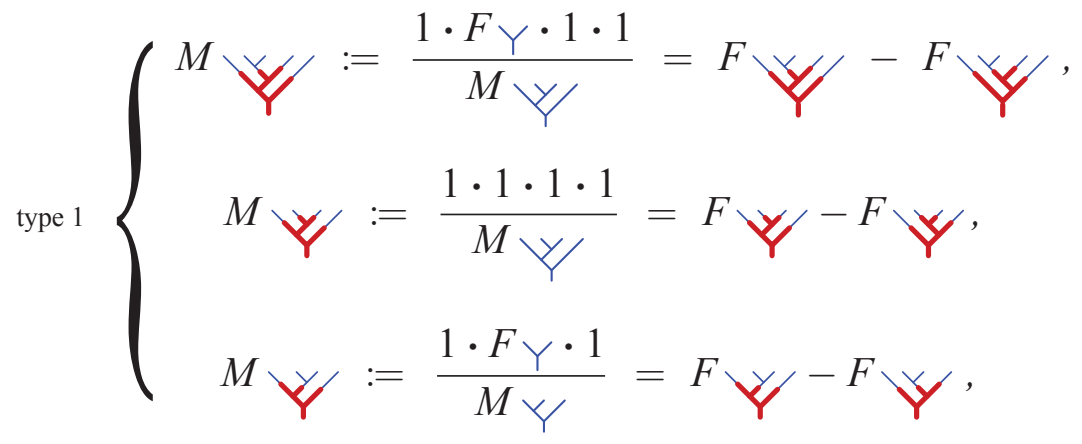

$$
\begin{aligned}
& \text { type 2 }\left\{M Y:=\frac{M Y}{1}=F Y Y-F Y Y\right. \text {. }
\end{aligned}
$$

Figure 16. Primitive elements of two types in $\mathcal{P}$ Sym.

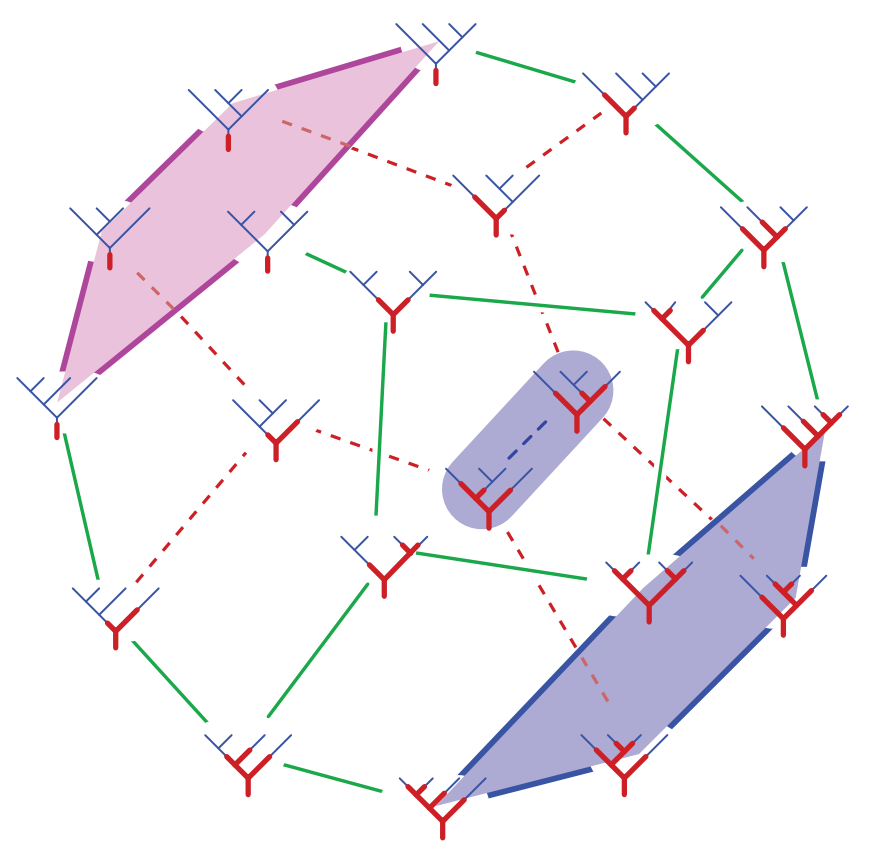

Figure 17. The multiplihedron lattice $\mathcal{M}_{4}$ showing the three subintervals that yield primitives via Möbius transformation.

\section{REFERENCES}

1. Marcelo Aguiar and Swapneel Mahajan, Monoidal functors, species and Hopf algebras, CRM Monograph Series, vol. 29, American Mathematical Society, Providence, RI, 2010.

2. Marcelo Aguiar and Frank Sottile, Structure of the Malvenuto-Reutenauer Hopf algebra of permutations, Adv. Math. 191 (2005), no. 2, 225-275. 
3. - Structure of the Loday-Ronco Hopf algebra of trees, J. Algebra 295 (2006), no. 2, $473-511$.

4. J. M. Boardman and R. M. Vogt, Homotopy invariant algebraic structures on topological spaces, Lecture Notes in Mathematics, Vol. 347, Springer-Verlag, Berlin, 1973.

5. Michael P. Carr and Satyan L. Devadoss, Coxeter complexes and graph-associahedra, Topology Appl. 153 (2006), no. 12, 2155-2168. MR MR2239078 (2007c:52012)

6. Frédéric Chapoton, Algèbres de Hopf des permutahèdres, associahèdres et hypercubes, Adv. Math. 150 (2000), no. 2, 264-275.

7. Stefan Forcey, Convex hull realizations of the multiplihedra, Topology Appl. 156 (2008), no. 2, $326-347$.

8. , Quotients of the multiplihedron as categorified associahedra, Homology, Homotopy Appl. 10 (2008), no. 2, 227-256.

9. Stefan Forcey, Aaron Lauve, and Frank Sottile, Hopf structures on the multiplihedra, SIAM J. Discrete Math. 24 (2010), 1250-1271.

10. (2011), 363-374.

11. Stefan Forcey and Derriell Springfield, Geometric combinatorial algebras: cyclohedron and simplex, J. Alg. Combin. 32 (2010), no. 4, 597-627.

12. André Joyal, Foncteurs analytiques et especes de structures, Combinatoire enumerative (Montreal, Quebec, 1985), Lecture Notes in Math., vol. 1234, Springer, Berlin, 1986, pp. 126-159.

13. Jean-Louis Loday and María O. Ronco, Hopf algebra of the planar binary trees, Adv. Math. 139 (1998), no. 2, 293-309.

14. Nathan Reading, Lattice congruences, fans and Hopf algebras, J. Combin. Theory Ser. A 110 (2005), no. 2, 237-273.

15. - From the tamari lattice to cambrian lattices and beyond, Associahedra, Tamari Lattices and Related Structures: Tamari Memorial Festschrift (J. Marcel J. Stasheff F. MllerHoissen, J. Pallo, ed.), Progress in Mathematics, vol. 299, 2012, pp. 299-322.

16. Maria Ronco, Generalized tamari order, Associahedra, Tamari Lattices and Related Structures: Tamari Memorial Festschrift (J. Marcel J. Stasheff F. Mller-Hoissen, J. Pallo, ed.), Progress in Mathematics, vol. 299, 2012, pp. 339-350.

17. Samson Saneblidze and Ronald Umble, Diagonals on the permutahedra, multiplihedra and associahedra, Homology Homotopy Appl. 6 (2004), no. 1, 363-411 (electronic).

18. James Stasheff, Homotopy associativity of H-spaces. I, II, Trans. Amer. Math. Soc. 108 (1963), 275-292; ibid. 108 (1963), 293-312.

19. , H-spaces from a homotopy point of view, Lecture Notes in Mathematics, Vol. 161, Springer-Verlag, Berlin, 1970.

20. Andy Tonks, Relating the associahedron and the permutohedron, Operads: Proceedings of Renaissance Conferences (Hartford, CT/Luminy, 1995), Contemp. Math., vol. 202, Amer. Math. Soc., Providence, RI, 1997, pp. 33-36.

(S. Forcey) Department of Mathematics, The University of Akron, Akron, OH 443254002

E-mail address: sf34@uakron.edu

$U R L:$ http://www.math.uakron.edu/ ${ }^{\text {sf } 34 /}$ 